\title{
Synthesis and Purification of Silver Nanowires To Make Conducting Films with a Transmittance of $99 \%$
}

\author{
Bo Li, ${ }^{\dagger, \dagger}$ Shengrong Ye, ${ }^{\dagger}$ Ian E. Stewart, ${ }^{\dagger}$ Samuel Alvarez, ${ }^{\dagger}$ and Benjamin J. Wiley ${ }^{*} \dagger$ \\ ${ }^{\dagger}$ Department of Chemistry, Duke University, Durham, North Carolina 27708, United States \\ ${ }^{\ddagger}$ State Key Laboratory for Modification of Chemical Fibers and Polymer Materials, College of Materials Science and Engineering, \\ Donghua University, Shanghai 201620, China
}

Supporting Information

ABSTRACT: Metal nanowire (NW) networks have the highest performance of any solution-coatable alternative to ITO, but there is as yet no published process for producing NW films with optoelectronic performance that exceeds that of ITO. Here, we demonstrate a process for the synthesis and purification of Ag NWs that, when coated from an ink to create a transparent conducting film, exhibit properties that exceed that of ITO. The diameter, and thus optoelectronic performance, of Ag NWs produced by a polyol synthesis can be controlled by adjusting the concentration of bromide. Ag NWs with diameters of $20 \mathrm{~nm}$ and aspect ratios up to 2000 were obtained by adding $2.2 \mathrm{mM} \mathrm{NaBr}$ to a $\mathrm{Ag} \mathrm{NW}$ synthesis, but these NWs were contaminated by nanoparticles. Selective precipitation was used to purify the NWs, resulting in a transmittance improvement as large as $4 \%$. At $130.0 \Omega \mathrm{sq}^{-1}$, the transmittance of the

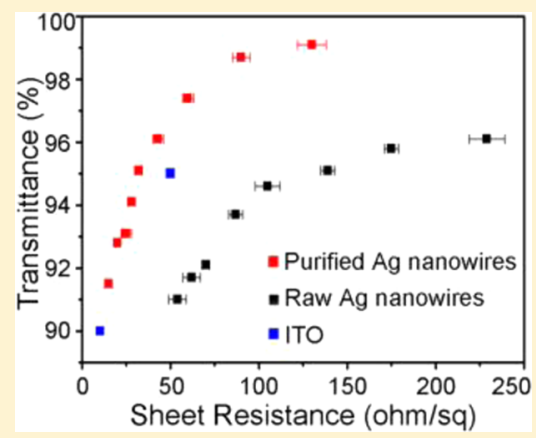
purified Ag NW film was $99.1 \%$.

KEYWORDS: silver nanowires, sodium bromide, selective precipitation, transparent conductor

$\mathrm{T}$ ransparent conductors (TCs) in large-area touch screens, thin-film solar cells, and organic light emitting diodes (OLEDs) are currently made from a sputtered film of indium tin oxide (ITO) due to its high transmittance (95\%T) at low sheet resistances $\left(50 \Omega \mathrm{sq}^{-1}\right){ }^{1}$ However, the high-conductivity ITO used in these applications is particularly costly due to the slow coating rates involved with sputtering. This has motivated the search for solution-coatable alternatives to ITO that can be coated at high speeds and give comparable performance. There are several solution-coatable alternatives to ITO, including carbon nanotubes, ${ }^{2-4}$ graphene, ${ }^{5,6}$ conducting polymers, ${ }^{7,8}$ silver nanowires (Ag NWs), ${ }^{9-12}$ and copper nanowires $(\mathrm{Cu}$ NWs), ${ }^{13-15}$ but only commercially available $\mathrm{Ag}$ NWs have demonstrated optoelectronic performance that exceeds that of ITO. ${ }^{16}$ The processes required for production of metal NWs that, when coated onto a substrate, have optoelectronic performance that exceeds that of ITO have not been reported.

The performance of $\mathrm{Ag} \mathrm{NW}$-based TCs generally increases with decreasing diameter and increasing NW aspect ratio due to the fact that thin NWs scatter less light, and increasing aspect ratio decreases the number of high-resistance nanowirenanowire contacts in the film. ${ }^{17,18}$ Although there have been many reported syntheses seeking to produce longer, thinner Ag NWs, none have demonstrated film performance that exceeds that of ITO. ${ }^{9,12,19} \mathrm{Ag}$ NWs with a mean diameter of $20 \mathrm{~nm}$ and lengths up to $20 \mu \mathrm{m}$ were prepared by a high-pressure polyol method. ${ }^{12}$ These nanowires were used to produce films with a sheet resistance of $40 \Omega \mathrm{sq}^{-1}$ at a transmittance of $88 \%$, performance below that of ITO. Lee and co-workers have developed a successive multistep growth method to obtain $\mathrm{Ag}$
NWs with an average length of $95.1 \mu \mathrm{m}$ and diameter of 160 nm. Although such long NWs exhibited optoelectronic performance that exceeds that of shorter NWs, their diameters are too large to match the properties of ITO. ${ }^{11}$ Thus, there remains a need for a synthesis of Ag NWs that are both very thin ( $\sim 20 \mathrm{~nm}$ in diameter) and very long ( $>50 \mu \mathrm{m}$ in length) so as to achieve ITO-level performance.

However, it is not enough to obtain Ag NWs with the right dimensions; they must also be highly pure and uncontaminated by the presence of nanoparticles. Nanoparticle impurities dramatically decrease the electrical conductivity and transparency of NW networks. ${ }^{20,21}$ Given the fact that the polyol methods most widely used for production of $\mathrm{Ag}$ NWs inevitably produce nanoparticles as a side product, this remains an important issue for the production of high-purity $\mathrm{Ag}$ NWs. ${ }^{12,19,22-24}$ The traditional purification process of performing many tedious cycles of centrifugation is time-consuming and, at least for the authors, has not produced Ag NWs with sufficient purity. ${ }^{10,22}$ Pradel and co-workers developed a crossflow filtration method to purify NWs, but the success of this method depends on many parameters, such as NW size, pore size of the fiber membrane, flow rate, and pressure, and is not easily scalable. ${ }^{21}$ Thus, there remains a need for a simple, convenient, and scalable method for separating nanoparticles from NWs.

Received: June 30, 2015

Revised: September 11, 2015

Published: September 21, 2015 


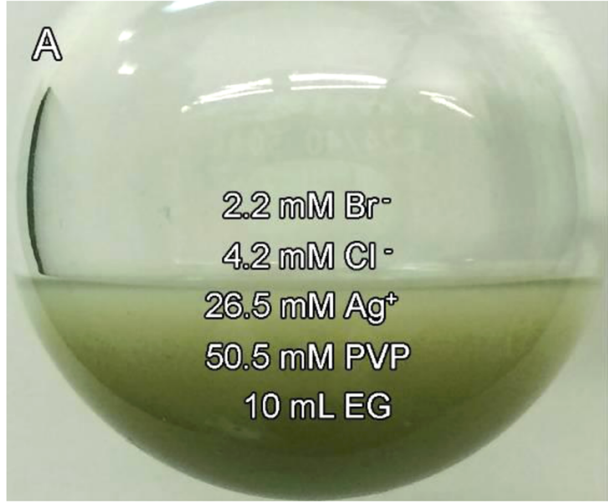

$\mathrm{B}$

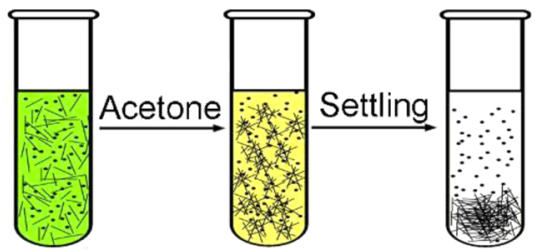

C

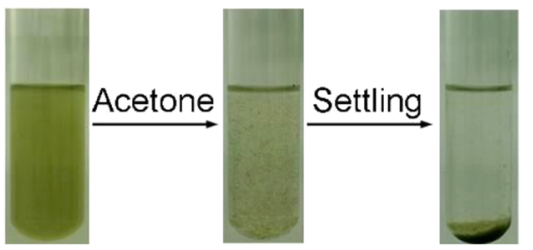

Figure 1. (A) Camera picture of the reaction flask after the growth of $\mathrm{Ag} N W s$ at $170{ }^{\circ} \mathrm{C}$ for $1 \mathrm{~h}$. (B) Scheme demonstrating the process for purification of Ag NWs. (C) Pictures showing the stages of the purification process.
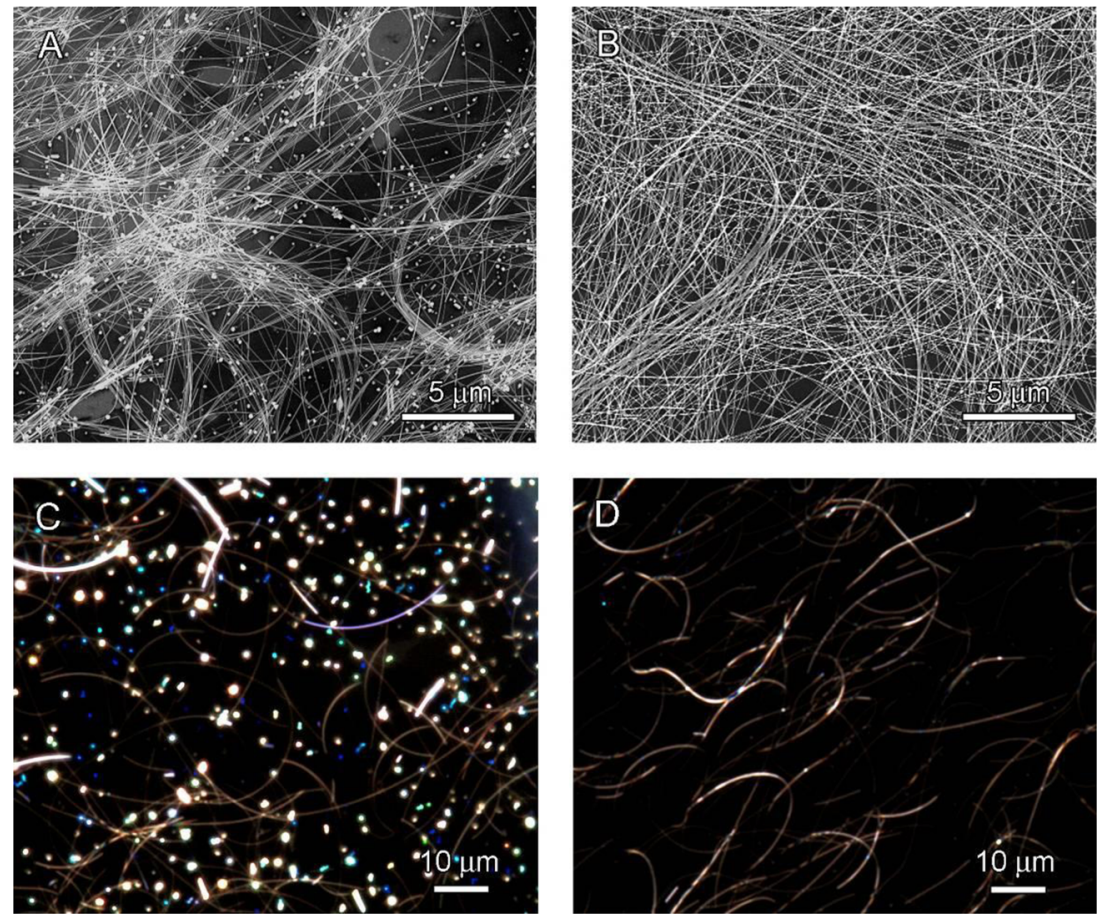

Figure 2. SEM images of Ag nanowires before (A) and after (B) purification. Dark field optical microscope images of Ag nanowires before (C) and after (D) purification.

Here, we show that the diameter of Ag NWs in a polyol synthesis can be controlled by adjusting the concentration of bromine. Ag NWs with diameters of $20 \mathrm{~nm}$ and aspect ratios up to 2000 were obtained by adding $2.2 \mathrm{mM} \mathrm{NaBr}$ to a $\mathrm{Ag} \mathrm{NW}$ synthesis. We also demonstrate a simple selective precipitation process to purify the Ag NWs. Purified Ag NWs were transferred to an ethyl cellulose-based ink formulation and coated onto glass with a Meyer rod to produce films with a transmittance of $99.1 \%$ at $130 \Omega \mathrm{sq}^{-1}$.

Ag NWs were synthesized through a modified polyol process. $^{25,26}$ To start, three stock solutions were prepared: (A) $220.0 \mathrm{mM} \mathrm{NaBr}$, (B) $210.0 \mathrm{mM} \mathrm{NaCl}$, and (C) $505.0 \mathrm{mM}$ PVP $(56.0 \mathrm{~g} / \mathrm{L}, \mathrm{MW}=130,000)$ in ethylene glycol $(\mathrm{EG}, \mathrm{J} . \mathrm{T}$. Baker). Next, $7.7 \mathrm{~mL}$ of EG, $0.1 \mathrm{~mL}$ of solution $\mathrm{A}, 0.2 \mathrm{~mL}$ of solution $\mathrm{B}, 1.0 \mathrm{~mL}$ of solution $\mathrm{C}$, and $1.0 \mathrm{~mL}$ of freshly prepared $265.0 \mathrm{mM} \mathrm{AgNO}_{3}$ in $\mathrm{EG}$ were added into a $50 \mathrm{~mL}$ round-bottom flask placed in an oil bath at room temperature. The flask was stirred vigorously for $30 \mathrm{~min}$, and then heated to
$170{ }^{\circ} \mathrm{C}$ with stirring for $15 \mathrm{~min}$. During heating, nitrogen gas was bubbled through the reaction solution. Once it reached 170 ${ }^{\circ} \mathrm{C}$, the flask was capped and allowed to react for $1 \mathrm{~h}$ without stirring. After $1 \mathrm{~h}$, the flask was removed from the oil bath and $30 \mathrm{~mL}$ of water was added to the reaction solution to cool it to room temperature, thereby stopping the reaction. Figure $1 \mathrm{~A}$ shows a picture of the reaction flask after $\mathrm{Ag} \mathrm{NW}$ growth.

After the synthesis, the Ag NWs were purified by selective precipitation (Figure 1B and C). This process simply involved the slow addition of acetone to the mixture of the reaction solution and water. Because the Ag NWs were coated with PVP, and PVP is not soluble in acetone, the Ag NWs aggregated and settled to the bottom of the vessel. As the aggregation involved a change in the color of the suspension from green to yellow (Figure 1C), it could be observed visually. This color change occurred when $80-160 \mathrm{~mL}$ of acetone was added, at which point no additional acetone was added and the NWs settled to the bottom of the vessel. The aggregated NWs 

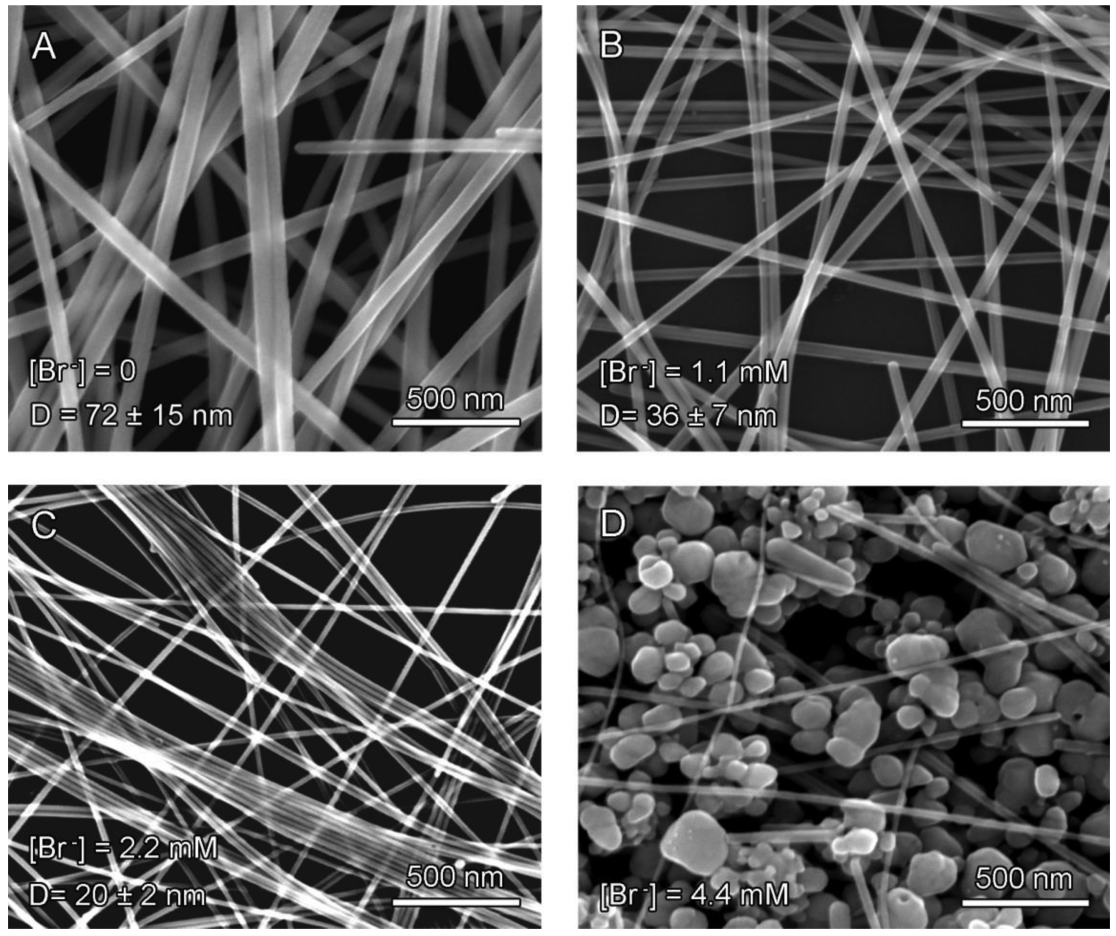

Figure 3. Typical high-resolution SEM images of purified products obtained from different concentrations of NaBr: (A) 0, (B) 1.1, (C) 2.2, and (D) 4.4 mM. The concentrations of $\mathrm{AgNO}_{3}, \mathrm{PVP}$, and $\mathrm{NaCl}$ in each of these reactions was $26.5,50.5$, and $4.2 \mathrm{mM}$, respectively, and the reaction temperature was $170{ }^{\circ} \mathrm{C}$.

settled to the bottom of the cylinder within $10 \mathrm{~min}$, at which point the supernatant containing short NWs and nanoparticles was removed with a pipet. The aggregated NWs were redispersed in $20 \mathrm{~mL}$ of DI water containing $0.5 \mathrm{wt} \%$ PVP. The redispersed NWs were purified again by adding $40-80 \mathrm{~mL}$ of acetone, allowing the NWs to settle for $10 \mathrm{~min}$, and removing the supernatant. NWs with high purity were obtained after 2-4 cycles of this purification process.

Figure 2 shows the images of Ag NWs with diameters of 20 $\pm 2 \mathrm{~nm}$ and lengths of $40 \pm 15 \mu \mathrm{m}$ before and after four cycles of selective precipitation (see Figure S1 in Supporting Information for histograms). There is clearly a dramatic decrease in the number of particles after the purification process. This same process could be applied to purify nanowires with larger diameters without modification (Figures S2 and S3 in Supporting Information).

Halide ions play an important role in the polyol synthesis of Ag NWs. ${ }^{9,19,25-28}$ We varied the concentration of $\mathrm{NaBr}$ added to the synthesis to gain additional insight into how it affects nanowire diameter. When there was no bromine present in the polyol synthesis, it produced $\mathrm{Ag}$ NWs with a mean diameter of $72 \pm 15 \mathrm{~nm}$ and lengths of $63 \pm 17 \mu \mathrm{m}$ (Figure $3 \mathrm{~A}$ and Supporting Information Figure S2). Addition of $1.1 \mathrm{mM} \mathrm{NaBr}$ reduced the diameter of the NWs to $36 \pm 7 \mathrm{~nm}$ (Figure $3 \mathrm{~B}$ and Supporting Information Figure S3), with lengths of $48 \pm 15$ $\mu \mathrm{m}$. Addition of $2.2 \mathrm{mM} \mathrm{NaBr}$ reduced the diameter of the NWs further to $20 \pm 2 \mathrm{~nm}$ (Figure 3C and Supporting Information Figure S1), with lengths of $40 \pm 13 \mu \mathrm{m}$. Further increasing the concentration of $\mathrm{NaBr}$ to $4.4 \mathrm{mM}$ resulted in many more nanoparticles in the reaction product (Figure 3D). If $8.8 \mathrm{mM} \mathrm{NaBr}$ was added, the main products were nanoparticles (Supporting Information Figure S4).

Experimental results presented in Table S1 in Supporting Information suggest that the decrease in diameter upon addition of $\mathrm{NaBr}$ was primarily caused by an increase in the number of nucleation events, with a smaller contribution from a decrease in the conversion of $\mathrm{Ag}^{+}$to Ag. Increasing the concentration of $\mathrm{NaBr}$ from 0 to $2.2 \mathrm{mM}$ increased the number of NWs produced in the reaction by a factor of 13 , from $5.6 \times$ $10^{12}$ to $73.5 \times 10^{12}$, whereas the average nanowire volume decreased by a factor of 20 , primarily due to the decrease in nanowire diameter. The increase in the number of NWs produced by the reaction suggests that there was an increase in the number of nucleation events upon addition of $\mathrm{NaBr}$. We attribute this increase in nucleation events to the presence of a silver halide nanoparticle precipitate. In our experiment, the chemicals were mixed at room temperature before heating. In the absence of $\mathrm{NaCl}$ or $\mathrm{NaBr}$, the reaction solution was clear after mixing at room temperature (Figure S5A in Supporting Information). In contrast, the solution with $\mathrm{NaCl}$ was a translucent white, and the solution with $\mathrm{NaBr}$ was a more opaque white or pale yellow (Figures S5B-D), indicating the formation of $\mathrm{AgCl}$ and $\mathrm{AgBr}$. The extinction spectrum peak intensity for solutions with $\mathrm{NaBr}$ was $\sim 2.4$ times greater than for $\mathrm{NaCl}$ alone (Figure $\mathrm{S} 6$ in the Supporting Information), indicating more precipitate formed after addition of $\mathrm{NaBr}$. Figure S7 in Supporting Information shows TEM images of the silver halide nanoparticles and histograms of the nanoparticle diameters. TEM imaging of the precipitate showed that the diameter of the $\mathrm{AgCl}$ nanoparticles $(180 \pm 57 \mathrm{~nm})$ was about 5 times greater than the diameter of the $\mathrm{AgBr}$ nanoparticles (35 $\pm 13 \mathrm{~nm}$ ). When both the nanoparticle size and change in absorption were taken into account, the number of silver halide nanoparticles increased by $\sim 12$ times after addition of $\mathrm{NaBr}$ relative to the number of nanoparticles present with $\mathrm{NaCl}$ alone. Both $\mathrm{AgBr}$ and $\mathrm{AgCl}$ have been shown to act as heterogeneous nucleants for growth of Ag NWs. ${ }^{9,29,30}$ Thus, the 13-fold increase in the number of nanowires upon addition 

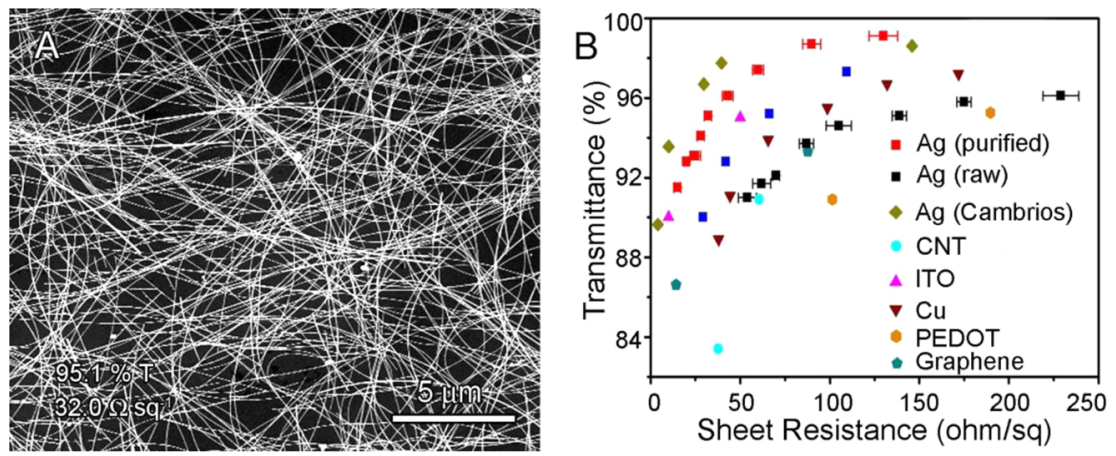

Figure 4. (A) Typical SEM image of a Ag NW film. (B) Plot of specular transmittance $(\lambda=550 \mathrm{~nm})$ vs sheet resistance for Ag NW films before and after purification. Error bars show one standard deviation for five measurements. The performance of ITO, Ag NWs, ${ }^{16} \mathrm{CNT},{ }^{33} \mathrm{Cu} \mathrm{NWs},{ }^{34} \mathrm{PEDOT}$, and graphene ${ }^{6}$ are shown for comparison.

of $\mathrm{NaBr}$ may have been caused by the $\sim 12$-fold increase in the number of silver halide nanoparticles that could serve as heterogeneous nuclei.

The remaining contribution to the 20 -fold decrease in NW volume can be attributed to the decrease in the \% conversion of $\mathrm{Ag}^{+}$to $\mathrm{Ag}$ from 77.7 to $58.6 \%$ upon addition of $2.2 \mathrm{mM} \mathrm{NaBr}$ to the reaction. At present it is not entirely clear why the presence of $\mathrm{NaBr}$ reduced the reduction rate of $\mathrm{Ag}^{+}$to $\mathrm{Ag}$. $\mathrm{Hu}$ et al. have suggested that the addition of $\mathrm{Br}^{-}$and subsequent formation of $\mathrm{AgBr}$ decreased the concentration of dissociated $\mathrm{Ag}^{+}$ions and thereby reduced the reduction rate of $\mathrm{Ag}^{+}$, leading to the formation of thinner nanowires. ${ }^{9}$ An alternative hypothesis is that $\mathrm{Br}^{-}$adsorbed on the surfaces of the $\mathrm{Ag}$ NWs reduced the rate of atomic addition to the Ag NWs. ${ }^{31}$

Reactions were also carried out using PVP with different molecular weights to see if the molecular weight was an important factor in determining NW diameter. When the molecular weight of PVP was $55 \mathrm{~K}$ instead of $130 \mathrm{~K}$, the diameter of the NWs was $25 \pm 2 \mathrm{~nm}$ (Figures S8A and B in the Supporting Information), slightly larger than the $20 \pm 2 \mathrm{~nm}$ obtained for PVP with a molecular weight of $130 \mathrm{~K}$. The diameter could be decreased to $21 \mathrm{~nm}$ in the presence of 2.75 $\mathrm{mM} \mathrm{NaBr}$ with a molecular weight of $\mathrm{PVP}=55 \mathrm{~K}$ (Figure S9 in the Supporting Information). If the PVP molecular weight was reduced to $29 \mathrm{~K}$, nanoparticles were the dominant product (Figure S8C in the Supporting Information). Thus, as demonstrated previously in similar reactions, the molecular weight of PVP is important in determining the yield of NWs relative to nanoparticles, but it does not have a very significant effect on nanowire diameter. ${ }^{22,24,32}$

Purified Ag NWs were transferred into an ink containing ethyl cellulose, ethyl alcohol, isopropyl alcohol, ethyl acetate, pentyl acetate, and toluene for coating with a Meyer rod. We have previously used a nitrocellulose-based ink for making $\mathrm{Cu}$ NW films, but this ink requires the use of acetone to dissolve nitrocellulose, and Ag NWs are not easily dispersed in acetone because they are coated with PVP (which is not soluble in acetone). Figure 4A shows a SEM image of a Ag NW film coated with a Meyer rod from the ethyl cellulose ink. Figure 4B shows a plot of specular transmittance (\%T) versus sheet resistance $\left(R_{\mathrm{s}}\right)$ for films of $20 \mathrm{~nm} \mathrm{Ag} \mathrm{NWs} \mathrm{before} \mathrm{and} \mathrm{after}$ purification, along with some of the best previous literature results for solution-coatable transparent conductors. The transmittance of films made from purified NWs was up to $4 \%$ greater than films made from NWs without purification. The performance of the purified NWs exceeds the performance of any other transparent conductor at sheet resistances $>75 \Omega$ $\mathrm{sq}^{-1}$. Only Ag NWs from Cambrios have higher values at lower sheet resistance. These results further demonstrate the importance of the aspect ratio, diameter, and purity on the performance of NW-based transparent conductors. ${ }^{1}$

In conclusion, the introduction of $\mathrm{NaBr}$ into a polyol synthesis enables the production of $\mathrm{Ag}$ NWs with aspect ratios $>2000$, and diameters of $20 \mathrm{~nm}$. The reduction in NW diameter obtained through the addition of $\mathrm{NaBr}$ was primarily due to an increase in the number of nucleation events, and secondarily due to a reduction in the \% conversion of $\mathrm{Ag}^{+}$to $\mathrm{Ag}$. The nanoparticles that inevitably contaminate the NWs produced by a polyol synthesis can be removed with a simple, scalable selective precipitation process. When coated from an ethyl cellulose-based ink to make a transparent conducting film, purified Ag NWs exhibited a transmittance of $99.1 \%$ at $130.0 \Omega$ $\mathrm{sq}^{-1}$. Purification of the NWs improved the transmittance at a given sheet resistance by as much as $4 \%$. Variations of the selective precipitation process can likely enable the purification of a wide variety of NWs. The synthesis and purification process demonstrated here is expected to be very useful for researchers in industry and academia seeking to produce NWbased films with performance that exceeds that of ITO.

\section{ASSOCIATED CONTENT}

\section{S Supporting Information}

The Supporting Information is available free of charge on the ACS Publications website at DOI: 10.1021/acs.nanolett.5b02582.

Detailed description of experimental methods and additional figures. (PDF)

\section{AUTHOR INFORMATION}

\section{Corresponding Author}

*E-mail: Benjamin.Wiley@duke.edu.

\section{Author Contributions}

B.L. and S.Y. contributed equally to this work.

Notes

The authors declare no competing financial interest.

\section{ACKNOWLEDGMENTS}

This work was supported by NSF's Research Triangle MRSEC (DMR-1121107), NSF grant no. ECCS-1344745 and an NSF CAREER award (DMR-1253534). B.L. was supported by the Fundamental Research Fund for the Central Universities and a fellowship from the China Scholarship Council. I.E.S. was 
supported by a fellowship through the Duke graduate program in nanoscience.

\section{REFERENCES}

(1) Ye, S.; Rathmell, A. R; Chen, Z.; Stewart, I. E.; Wiley, B. J. Adv. Mater. 2014, 26, 6670.

(2) Zhang, D.; Ryu, K.; Liu, X.; Polikarpov, E.; Ly, J.; Tompson, M. E.; Zhou, C. Nano Lett. 2006, 6, 1880.

(3) Wu, Z.; Chen, Z.; Du, X.; Logan, J. M.; Sippel, J.; Nikolou, M.; Kamaras, K.; Reynolds, J. R.; Tanner, D. B.; Hebard, A. F.; Rinzler, A. G. Science 2004, 305, 1273

(4) Geng, H. Z.; Kim, K. K.; So, K. P.; Lee, Y. S.; Chang, Y.; Lee, Y.

H. J. Am. Chem. Soc. 2007, 129, 7758.

(5) Wassei, J. K.; Kaner, R. B. Mater. Today 2010, 13, 52.

(6) Lee, J. H.; Shin, D. W.; Makotchenko, V. G.; Nazarov, A. S.; Fedorov, V. E.; Kim, Y. H.; Choi, J. Y.; Kim, J. M.; Yoo, J. B. Adv. Mater. 2009, 21, 4383.

(7) Elschner, A.; Lovenich, W. MRS Bull. 2011, 36, 794.

(8) Na, S. I.; Kim, S. S.; Jo, J.; Kim, D. Y. Adv. Mater. 2008, 20, 4061.

(9) Hu, L.; Kim, H. S.; Lee, J.; Peumans, P.; Cui, Y. ACS Nano 2010, 4, 2955.

(10) Mayousse, C.; Celle, C.; Moreau, E.; Mainguet, J. F.; Carella, A.; Simonato, J. P. Nanotechnology 2013, 24, 215501.

(11) Lee, J.; Lee, P.; Lee, H.; Lee, D.; Lee, S. S.; Ko, S. H. Nanoscale 2012, 4, 6408.

(12) Lee, E. J.; Chang, M.-H.; Kim, Y.-S.; Kim, J.-Y. APL Mater. 2013, 1,042118 .

(13) Guo, H.; Lin, N.; Chen, Y.; Wang, Z.; Xie, Q.; Zheng, T.; Gao, N.; Li, S.; Kang, J.; Cai, D.; Peng, D. L. Sci. Rep. 2013, 3, 2323.

(14) Rathmell, A. R.; Bergin, S. M.; Hua, Y. L.; Li, Z. Y.; Wiley, B. J. Adv. Mater. 2010, 22, 3558.

(15) Rathmell, A. R.; Wiley, B. J. Adv. Mater. 2011, 23, 4798.

(16) Cambrios Technologies. http://www.cambrios.com/ (accessed May 2015).

(17) Mutiso, R. M.; Sherrott, M. C.; Rathmell, A. R.; Wiley, B. J.; Winey, K. I. ACS Nano 2013, 7, 7654.

(18) Bergin, S. M.; Chen, Y. H.; Rathmell, A. R.; Charbonneau, P.; Li,

Z. Y.; Wiley, B. J. Nanoscale 2012, 4, 1996.

(19) Chang, Y.-H.; Lu, Y.-C.; Chou, K.-S. Chem. Lett. 2011, 40, 1352.

(20) Tao, A.; Sinsermsuksakul, P.; Yang, P. Angew. Chem., Int. Ed. 2006, 45, 4597.

(21) Pradel, K. C.; Sohn, K.; Huang, J. Angew. Chem., Int. Ed. 2011 , $50,3412$.

(22) Sun, Y. G.; Yin, Y. D.; Mayers, B. T.; Herricks, T.; Xia, Y. N. Chem. Mater. 2002, 14, 4736.

(23) Sun, Y. G.; Xia, Y. N. Adv. Mater. 2002, 14, 833.

(24) Zhu, J. J.; Kan, C. X.; Wan, J. G.; Han, M.; Wang, G. H. J. Nanomater. 2011, 2011, 1.

(25) Wiley, B. J.; Sun, Y. G.; Xia, Y. N. Langmuir 2005, 21, 8077.

(26) Wiley, B. J.; Sun, Y. G.; Xia, Y. N. Acc. Chem. Res. 2007, 40, 1067.

(27) Wiley, B. J.; Herricks, T.; Sun, Y. G.; Xia, Y. N. Nano Lett. 2004, 4, 1733.

(28) Wiley, B. J.; Im, S. H.; Li, Z. Y.; McLellan, J.; Siekkinen, A.; Xia, Y. N. J. Phys. Chem. B 2006, 110, 15666.

(29) Liu, S.; Yue, J.; Gedanken, A. Adv. Mater. 2001, 13, 656.

(30) Schuette, W. M.; Buhro, W. E. ACS Nano 2013, 7, 3844.

(31) Foresti, M. L.; Kobayashi, H.; Pezzatini, G.; Guidelli, R. J. Chem. Soc., Faraday Trans. 1996, 92, 3747.

(32) Zeng, X.; Zhou, B.; Gao, Y.; Wang, C.; Li, S.; Yeung, C. Y.; Wen, W. Nanotechnology 2014, 25, 495601.

(33) Hecht, D. S.; Heintz, A. M.; Lee, R.; Hu, L.; Moore, B.; Cucksey, C.; Risser, S. Nanotechnology 2011, 22, 075201.

(34) Ye, S.; Rathmell, A. R.; Stewart, I. E.; Ha, Y. C.; Wilson, A. R.; Chen, Z.; Wiley, B. J. Chem. Commun. 2014, 50, 2562. 